\title{
Nestmate recognition and levels of aggression are not altered by changes in genetic diversity in a socially polymorphic ant
}

\author{
HERVÉ ROSSET, TANJA SCHWANDER \& MICHEL CHAPUISAT \\ Department of Ecology and Evolution, University of Lausanne \\ (Received 24 July 2006; initial acceptance 21 September 2006; \\ final acceptance 3 November 2006; published online 31 August 2007; MS. number: 9059)
}

\begin{abstract}
The ability to distinguish nestmates from foreign individuals is central to the functioning of insect societies. In ants, workers from multiple-queen colonies are often less aggressive than workers from singlequeen ones. In line with this observation, it has been hypothesized that workers from multiple-queen colonies have less precise recognition abilities than workers from single-queen ones because their colonies contain genetically more diverse individuals, which results in a broader template of recognition cues. Here, we assessed the impact of social structure (queen number) variation on nestmate recognition and aggression in a large population of the socially polymorphic ant Formica selysi. We staged unilateral aggression tests on the nest surface. Workers from single- and multiple-queen colonies had good nestmate recognition ability and did not differ significantly in their level of aggression towards foreign, immobilized workers (cue-bearers). In particular, workers from multiple-queen colonies efficiently recognized non-nestmates despite the higher genetic diversity in their colony. Cue-bearers from single- and multiple-queen colonies elicited similar reactions. However, the level of aggression was higher between than within social forms, suggesting that workers detect a signal that is specific to the colony social structure. Finally, the level of aggression was not correlated with the genetic distance between colonies. Overall, we found no evidence for the hypothesis that the presence of multiple breeders in the same colony decreases recognition abilities and found no simple relationship between genetic diversity and aggression level.
\end{abstract}

(c) 2007 The Association for the Study of Animal Behaviour. Published by Elsevier Ltd. All rights reserved.

Keywords: aggressive behaviour; Formica selysi; kin recognition; nestmate discrimination; social evolution; social structure

The ability to recognize self from nonself is central to the functioning of many biological systems, from multicellular organisms to complex societies. In the social insects, individuals group into colonies that form the basic functional social units (Crozier \& Pamilo 1996). Members of social insect colonies usually distinguish colony members from nonmembers, which is essential for maintaining the integrity of the colony and ensuring that worker's altruism is directed towards related individuals (Hamilton 1964; Wilson 1971; Crozier \& Pamilo 1996).

Recognition systems involve two categories of participants, one that discriminates and the other that is discriminated. As proposed by Liebert \& Starks (2004), we call these participants evaluators and cue-bearers, which correspond to actors and recipients in kin selection

Correspondence: M. Chapuisat, Department of Ecology and Evolution, Biophore, UNIL-Sorge, University of Lausanne, 1015 Lausanne, Switzerland (email: michel.chapuisat@unil.ch). terminology, or receivers and signallers in signalling theory. Recognition systems rely on three distinct components: the expression of cues by the cue-bearer, the perception of cues by the evaluator, and the action taken by the evaluator, which depends on internal decision rules (Sherman et al. 1997; Liebert \& Starks 2004).

In social insects, individuals use chemical cues to distinguish nestmates from non-nestmates (Breed \& Bennett 1987; Hölldobler \& Wilson 1990). Cuticular hydrocarbons are major nestmate recognition cues in many species (Lahav et al. 1999; Wagner et al. 2000; Ruther et al. 2002; Breed et al. 2004; Buchwald \& Breed 2005; Howard \& Blomquist 2005; Ozaki et al. 2005). Recognition cues can be endogenous and have a strong genetic component (Greenberg 1979; Adams 1991; Crozier \& Pamilo 1996; Beye et al. 1998 ), or can be acquired from the environment (Downs \& Ratnieks 1999; Liang \& Silverman 2000; Buczkowski \& Silverman 2006). They are commonly transferred among nestmates by trophallaxis (the oral exchange of liquid food), allogrooming and passive contact, which results in 
a common colony odour, or Gestalt (Crozier \& Dix 1979; Soroker et al. 1995; Lahav et al. 1999; Boulay et al. 2000; Lenoir et al. 2001; Chapuisat et al. 2005).

The discrimination between nestmates and foreign individuals appears to be on the basis of a "phenotype matching' model (Waldman et al. 1988; Crozier \& Pamilo 1996; Sherman et al. 1997). Evaluators detect odour cues on cue-bearers and compare these profiles to an internal template representing the odour of their own colony, which they have learned (Breed \& Bennett 1987; Crozier $\&$ Pamilo 1996; Breed et al. 2004). The magnitude of the mismatch between the detected cues and the template triggers an appropriate behavioural response. Small differences often result in increased trophallaxis and allogrooming (Dahbi et al. 1999; Boulay et al. 2000; Lenoir et al. 2001; Chapuisat et al. 2005), whereas when the mismatch raises above some threshold, aggressive behaviour is elicited (Wilson 1971; Hölldobler \& Michener 1980; Reeve 1989).

There is a potential link between social structure and recognition ability. In ants, the number of queens per colony varies among species, among populations and even among colonies within the same population (Bourke \& Franks 1995; Crozier \& Pamilo 1996; Pamilo et al. 1997; Ross 2001). It has been hypothesized that the presence of multiple breeders in the same colony increases the diversity of heritable recognition cues and broadens both the mix of cues borne by workers and the internal template characterizing the colony (Hölldobler \& Wilson 1977; Breed \& Bennett 1987; Vander Meer \& Morel 1998). Therefore, individuals from multiple-queen colonies might be less efficient at recognizing foreign cue-bearers than individuals from single-queen colonies. Workers from multiple-queen colonies may thus accept intruders with variable cue profiles, while being themselves rejected by individuals from genetically less diverse colonies, in particular, single-queen ones (Vander Meer \& Morel 1998; Tsutsui et al. 2003). Interestingly, a positive feedback loop might exist between queen number and recognition, as a decrease in recognition ability might facilitate the acceptance of unrelated new queens, thus further increasing the genetic diversity within colonies and decreasing the genetic variance among colonies.

Several studies have indeed reported that aggression towards foreign individuals is reduced in ant populations or species with multiple-queen colonies, as compared to populations or species with single-queen colonies (Bennett 1989; Morel et al. 1990; Sundström 1997). Moreover, a few species with very high queen number show little aggression among distant nests (Giraud et al. 2002; Holzer et al. 2006). These studies are consistent with the hypothesis that high intracolony genetic diversity results in lower discrimination ability and reduced aggression. However, they did not investigate the precise relationship between genetic diversity, recognition ability and aggression level.

Few studies have investigated the impact of within population variation in colony queen number or genetic diversity on the level of aggression towards non-nestmates. Two studies found evidence for higher aggression in ant colonies with less genetic diversity (Pirk et al. 2001;
Tsutsui et al. 2003). In contrast, colonies with single or multiple reproductive egg-layers had similar aggression levels in three other ant species (Crosland 1990; Stuart 1991; Satoh \& Hirota 2005). The latter studies suggest that differences in colony genetic diversity may not be a proximate cause of decreased aggression. However, these studies did not include genetic data and two of them involved species with unusual breeding systems for ants (Crosland 1990; Satoh \& Hirota 2005). Hence, more empirical data are needed to better understand how queen number, genetic diversity, recognition ability and aggression levels interplay.

The ant Formica selysi offers a good opportunity to further investigate the relationship between social structure, colony genetic diversity and aggression, because in our study population single- and multiple-queen colonies occur in close proximity in the same habitat, whereas there is no genetic differentiation between the social forms (Chapuisat et al. 2004). We staged unilateral aggression tests on the nest surface, in the field. We first tested if workers discriminate between nestmates and non-nestmates. We then investigated how variation in colony social structure and genetic diversity modulated aggression in a natural system. In particular, we examined if higher genetic diversity in multiple-queen colonies resulted in reduced discrimination ability and lower levels of aggression towards non-nestmates.

\section{METHODS}

We studied a large population of $F$. selysi located along the river Rhône between Sierre and Susten in Switzerland (Chapuisat et al. 2004). The social structure of 112 colonies of this population had been unambiguously determined by genotyping at least eight workers from each colony at five to nine microsatellite markers (Chapuisat et al. 2004; Schwander et al. 2005). The population contains a mix of single- and multiple-queen colonies (63\% and $37 \%$, respectively) that often occur in close proximity. Our previous genetic analysis showed that F. selysi colonies occupy single nests, although some large nests might have multiple entrances (Chapuisat et al. 2004).

We designed a unilateral aggression assay that focuses on the behaviour of a single evaluator towards immobilized cue-bearers in field conditions. We placed a small arena on the nest surface $2 \mathrm{~h}$ prior to the beginning of the behavioural assay, so that ants could get familiar with it. The arena consisted of an open $6-\mathrm{cm}$ diameter petri dish with fluon-treated walls and sand from the nest at the bottom. The arena had a door and the focal workers (evaluators) entered freely into it. Cue-bearers were workers collected from an appropriate target colony and kept on ice until the start of the assay. Once a focal evaluator worker entered the arena, we shut the door. Two cuebearers were then immobilized with $\mathrm{CO}_{2}$ and placed on each side of the arena to increase the probability of encounters. We recorded the behaviour of the evaluator towards the cue-bearers for at least 2 min and until seven separate interactions or $1 \mathrm{~min}$ of interactions had taken place, whichever occurred first. 
Interactions were scored as follows: $0=$ ignore: short physical contact with no particular reaction; $1=$ antennation: the evaluator repeatedly touched the cue-bearer with its antennae; 2 = avoidance: the evaluator quickly retreated after contact with the cue-bearer; 3 = biting: the evaluator briefly bit an extremity of the cue-bearer, usually the legs or antennae; $4=$ biting and pulling: prolonged bites and traction on the cue-bearer. Individuals were used only once, and for each pair of nests the aggression tests were replicated five times with different individuals. An aggression index was calculated as the mean of the highest score obtained in each of the five replicates. A value of the aggression index of three or more thus reveals that aggression was high and consistent across the five replicates.

We selected pairs of nests that were separated from each other by at least $300 \mathrm{~m}$. Each colony in a pair served as a source of either evaluators or cue-bearers, respectively. The observers were blind with respect to the origin of cue-bearers. All behavioural tests were done in May 2002, which is a period of intense activity.

We first tested if workers discriminated between nestmates and non-nestmates. We assessed the reaction of evaluators from 11 colonies (six with a single queen and five with multiple queens) towards nestmate cue-bearers or cue-bearers coming from distant colonies. This experiment also permits to control that the $\mathrm{CO}_{2}$ immobilization of cuebearers does not prevent nestmate discrimination.

To study the effect of social structure variation on recognition and aggression, we selected 40 single- and 35 multiple-queen colonies. Single- and multiple-queen colonies (20 each) were chosen at random to serve as sources of evaluators, whereas the remaining 35 colonies served as sources of cue-bearers. Each of the 40 colonies providing evaluators was tested twice, once against evaluators from a single-queen colony, and once against evaluators from a multiple-queen colony. The impact of the social structure of evaluators and cue-bearers on aggression was tested in a two-way ANOVA. Because the aggression index is not a continuous variable, the statistical significance was obtained by generating the distribution of the null hypothesis with a permutation procedure (Manly 1997). To test for the effect of the social structure of the evaluators, the data were permuted between evaluators within cue-bearers of the same social structure. Similarly, to test for the effect of the social structure of the cue-bearers, the data were permuted between cue-bearers within evaluators of the same social structure. Finally, to test for the interaction, the data were permuted among assays independently of the social structure of the evaluators and cue-bearers (Manly 1997). For each scheme, 5000 permutations were carried out.

The genetic relatedness among worker nestmates was estimated from the microsatellite genotypes as described previously (Chapuisat et al. 2004). Additionally, we estimated the allelic richness per colony over five loci with a method correcting for sample size (El Mousadik \& Petit 1996), as implemented in the computer program FSTAT 2.9.3 (Goudet 1995, available at http://www2.unil.ch/ popgen/softwares/fstat.htm). We estimated the genetic distance between pairs of colonies that provided evaluators and cue-bearers with the Cavalli-Sforza chord measure
(Cavalli-Sforza \& Edwards 1967), as implemented in the computer program PHYLIP (J. Felsenstein 2005. Department of Genome Sciences, University of Washington, Seattle, available at http://evolution.genetics.washington. edu/phylip.html). This method estimates differences in allele frequencies between pairs of colonies independently of the level of genetic diversity within colonies.

\section{RESULTS}

Formica selysi workers discriminated nestmates from nonnestmates (Fig. 1). They reacted aggressively towards foreign cue-bearers, usually by biting them. In contrast, the reaction towards nestmate cue-bearers was generally nonaggressive, most often being limited to inspection and avoidance, with occasional short seizures of antennae and legs. The aggression scores might be inflated by the $\mathrm{CO}_{2}$ immobilization and because the highest score in each replicate was considered. However, the important point here is that the $\mathrm{CO}_{2}$ immobilization of cue-bearers did not prevent nestmate recognition, the aggression score towards cue-bearers originating from other colonies being significantly higher than the one towards nestmates that had been treated in exactly the same way (Fig. 1; exact Wilcoxon signed-ranks test: $Z=2.67, N=11, P=0.008$ ).

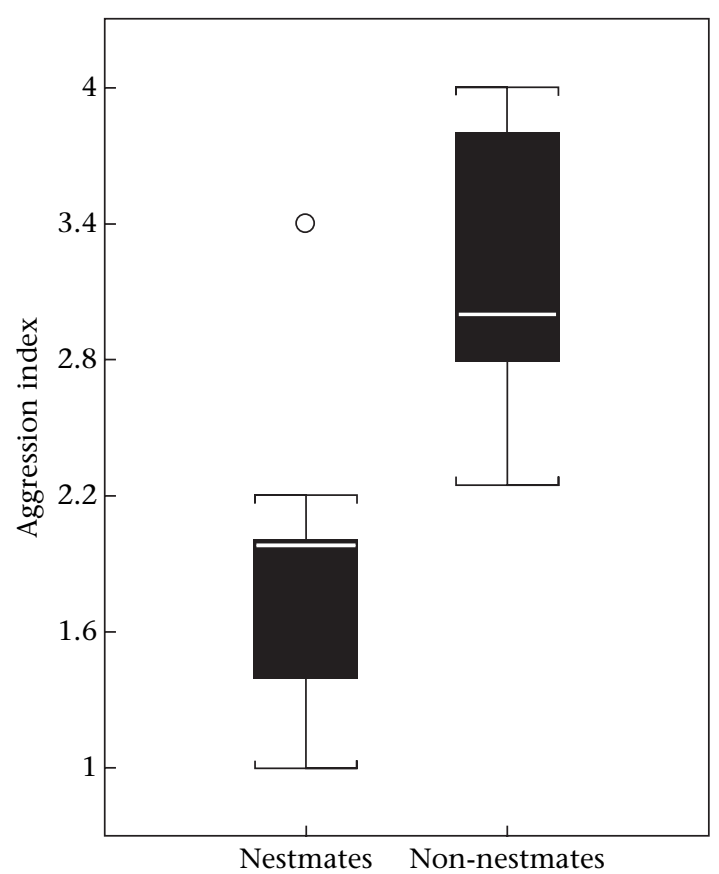

Figure 1. Aggression between workers from the same colony (nestmates) or from different colonies (non-nestmates). $N=11$ colonies (six single- and five multiple-queen ones), with five replicates of the aggression test per colony. The aggression index is the mean of the highest score per replicate, with scores of 0 for contacts followed by an absence of reaction, 1 for antennation, 2 for avoidance, 3 for brief biting and 4 for prolonged biting and pulling. The white bar is the median, the black box is the interquartile range and the whiskers correspond to the data range without outliers (points inferior to the first quartile minus 1.5 times the interquartile range or superior to the third quartile plus 1.5 times the interquartile range, indicated by open circles). 


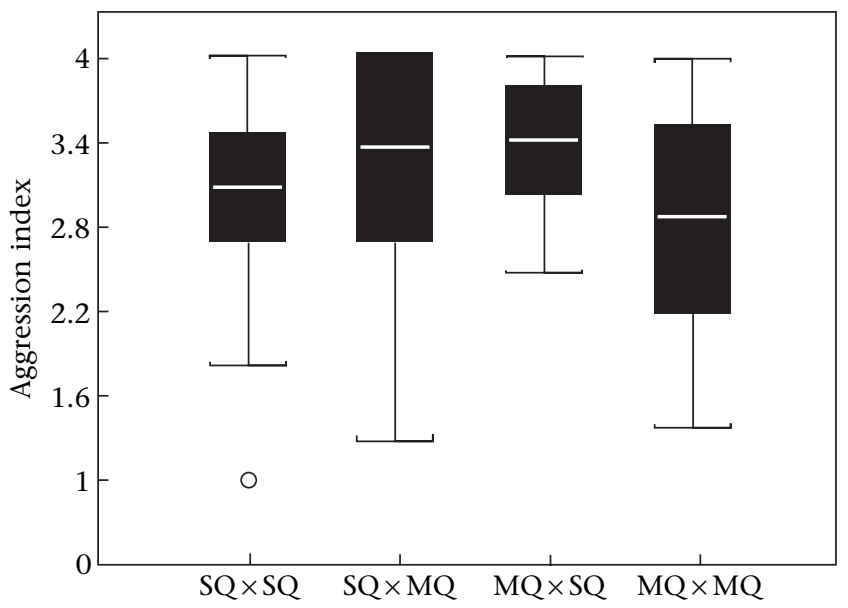

Figure 2. Aggression between workers from single-queen (SQ) and/ or multiple-queen $(\mathrm{MQ})$ colonies. For example, $\mathrm{SQ} \times \mathrm{MQ}$ indicates that the evaluator was from a single-queen colony and the cuebearers from a multiple-queen colony. $N=20$ pairs of colonies for each box, with five replicates of the aggression test per pair of colonies.

The impact of the social structure of the evaluators and cue-bearers on recognition and aggression was complex (Fig. 2). On average, the aggression level was not significantly different between evaluators coming from single- or multiple-queen colonies (Fig. 2; ANOVA, permutation test for the effect of the social structure of the evaluator: $P=0.74)$. Similarly, the social structure of the cue-bearers had no significant impact on the aggression level (Fig. 2; ANOVA, permutation test for the effect of the social structure of the cue-bearers: $P=0.41$ ). However, there was a significant interaction between the social structures of the evaluator and cue-bearer (Fig. 2; ANOVA, permutation test for the interaction: $P=0.008)$. Because of the significant interaction, we analysed aggression scores from single-and multiple-queen colonies separately with nonparametric tests. Evaluators from single-queen colonies tended to be more aggressive towards cue-bearers from multiple- than single-queen colonies (Wilcoxon twosample test: $\left.Z=1.86, N_{1}=N_{2}=20, P=0.06\right)$. Reciprocally, evaluators from multiple-queen colonies were significantly more aggressive towards cue-bearers from singlethan multiple-queen colonies (Wilcoxon two-sample test: $\left.Z=-2.1, N_{1}=N_{2}=20, P=0.035\right)$. Hence, evaluators from colonies of one type of social structure (singleor multiple-queen colony) were more aggressive towards cue-bearers from foreign colonies of the other type than towards cue-bearers from foreign colonies of their own type.

The genetic relatedness among worker nestmates was $0.14 \pm 0.11$ in the multiple-queen colonies and $0.80 \pm 0.05$ in the single-queen ones (mean $\pm \mathrm{SE}$ ). In line with this relatedness pattern, multiple-queen colonies had a significantly higher allelic richness than singlequeen ones (2.60 versus 1.63 , respectively; one-tailed $t$ test: $\left.t_{72}=18.81, P<0.001\right)$. As expected from the observed difference in relatedness and intracolony genetic diversity, the genetic distance between pairs of colonies

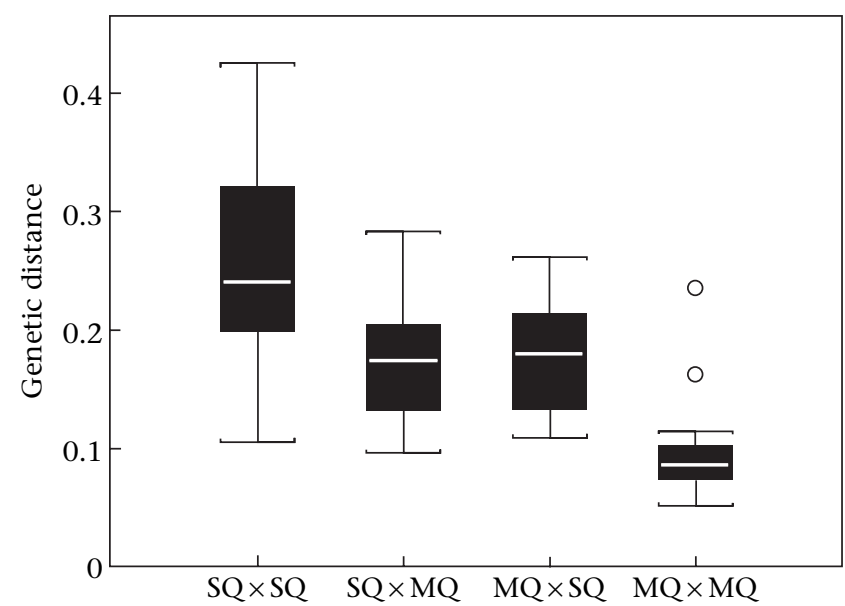

Figure 3. Cavalli-Sforza genetic distance between the pairs of singlequeen (SQ) and/or multiple-queen (MQ) colonies that were used in aggression tests. $N=20$ pairs of colonies for each box.

estimated with Cavalli-Sforza chord measure was significantly smaller for multiple-queen colonies than for single-queen ones (two-tailed $t$ test: $t_{38}=7.49, P<0.001$; Fig. 3). The aggression index and genetic distance between pairs of colonies were not significantly correlated over all colony pairs (Spearman rank correlation: $Z=1.08$, $N=80, P=0.28)$, nor within each class of interactions (Spearman rank correlations: single-queen $\times$ single-queen: $Z=0.58, \quad N=20, \quad P=0.56 ; \quad$ single-queen $\times$ multiplequeen: $\quad Z=1.51, \quad N=20, \quad P=0.13 ; \quad$ multiple-queen $\times$ single-queen: $Z=-1.11, \quad N=20, \quad P=0.27$; multiplequeen $\times$ multiple-queen: $Z=-0.18, N=20, P=0.85$ ).

\section{DISCUSSION}

Our behavioural assays performed on the nest surface in the field revealed that $F$. selysi workers distinguished nestmates from non-nestmates. Workers systematically attacked immobilized foreign workers, whereas they generally inspected or avoided immobilized nestmate workers, with rare signs of aggression. This result is in line with the good nestmate discrimination abilities found in many social insect species (Wilson 1971; Crozier \& Pamilo 1996).

We found no support for the hypothesis that a broader mix of genetically determined cues in multiple-queen colonies results in decreased aggression towards nonnestmates. The microsatellite analysis revealed that multiple-queen colonies were genetically more diverse than single-queen ones, and thus were likely to have broader templates of heritable cues. However, F. selysi workers from single- and multiple-queen colonies showed no significant difference in their level of aggression towards foreign cue-bearers. In particular, evaluators originating from multiple-queen colonies did not show significantly lower levels of aggression. Likewise, cue-bearers from single- and multiple-queen colonies did not elicit significantly different levels of aggression.

The similar level of aggression in single- and multiplequeen colonies suggests that an increase in queen number 
did not result in a loss of recognition ability in our study population and that there is no simple causal relationship between genetic diversity and discrimination ability. This result should, however, be interpreted with some caution, as aggression towards non-nestmates was used as a proxy for recognition ability. Electrophysiological studies of antennae activity (Ozaki et al. 2005) would be useful to disentangle the perception component from the action component in studies of nestmate recognition.

The lack of association between genetic diversity and aggression in our study population suggests that factors other than colony genetic diversity might cause the lower level of aggression that is often observed in ant populations with many queens per colony (Bennett 1989; Morel et al. 1990; Sundström 1997). One possibility is that the ecological conditions favouring high queen number, such as high population density, also select for reduced aggression (Stuart 1991; Holway et al. 1998; Giraud et al. 2002). Another possibility is that a loss of discrimination only occurs in species with a large number of queens per colony but not in populations in which colonies with single and multiple breeding females coexist (Crosland 1990; Stuart 1991). Again, studies aimed at disentangling recognition and aggression would permit to distinguish between the two hypotheses.

Interestingly, the level of aggression was significantly higher when the evaluators and cue-bearers came from colonies with different social structures. This pattern indicates that workers distinguish between individuals originating from single- and multiple-queen colonies, despite the complete lack of genetic differentiation between the two social forms above the colony level (Chapuisat et al. 2004). Workers apparently detect a signal that is specific to the colony social structure, which might be the overall diversity in odour cues, a characteristic mix of hydrocarbons (Breed et al. 2004), or other types of pheromones (Breed 1998; Vander Meer \& Alonso 2002; Hernandez et al. 2006). Alternatively, it is also possible that single- and multiple-queen colonies differ in subtle environmental or social factors that trigger increased levels of aggression. Hence, more experimental studies are needed to understand the causes of the increased aggression between social forms.

The level of aggression was not correlated with the genetic distance between colonies. As expected from the variation in queen number and genetic diversity, multiplequeen colonies were genetically closer than single-queen ones, whereas the genetic distance between colonies of different social structures was intermediate. This pattern further suggests that there is no simple relationship between the genetic similarity of colonies and the ability of workers to recognize non-nestmates. The decision rules used by workers to exclude non-nestmates are still poorly known, but our finding is in line with experiments in the honeybee that have found that guards use complex decision rules that depend on the concentration of multiple recognition cues present in the blend (Breed et al. 2004).

The lack of correlation between genetic distance and discrimination contrasts with four studies of Formica ants that have found such a correlation (Beye et al. 1997, 1998; Pirk et al. 2001; Holzer et al. 2006). In these studies both closely located and more distant nests were sampled, and the nests generally had multiple queens. Hence, recognition might be affected by local genetic similarity because of nest budding in multiple-queen colonies (e.g. Chapuisat et al. 1997), geographical variation in environmental cues (Buczkowski \& Silverman 2006) and possibly habituation (Langen et al. 2000). In contrast, we selected only pairs of distant colonies to avoid these effects and concentrate on whether the presence of multiple breeders in the same colony decreased recognition abilities. Overall, we found no evidence for such an effect, and no simple relationship between genetic diversity and aggression level.

\section{Acknowledgments}

We thank Dany Arsic for help in the field, Jérôme Goudet for help with statistics, Serge Aron, Robert L. Hammond, Laurent Keller, Pekka Pamilo and the anonymous referees for comments on the manuscript. This study was supported by grants from the Swiss National Science Foundation (nos 3100-61934 and 3100-108263).

\section{References}

Adams, E. S. 1991. Nest-mate recognition based on heritable odors in the termite Microcerotermes arboreus. Proceedings of the National Academy of Sciences, U.S.A., 88, 2031-2034.

Bennett, B. 1989. Nestmate recognition systems in a monogynouspolygynous species pair of ants (Hymenoptera: Formicidae). I. Worker and queen derived cues. Sociobiology, 16, 121-139.

Beye, M., Neumann, P. \& Moritz, R. F. A. 1997. Nestmate recognition and the genetic gestalt in the mound-building ant Formica polyctena. Insectes Sociaux, 44, 49-58.

Beye, M., Neumann, P., Chapuisat, M., Pamilo, P. \& Moritz, R. F. A. 1998. Nestmate recognition and the genetic relatedness of nests in the ant Formica pratensis. Behavioral Ecology and Sociobiology, 43, 67-72.

Boulay, R., Hefetz, A., Soroker, V. \& Lenoir, A. 2000. Camponotus fellah colony integration: worker individuality necessitates frequent hydrocarbon exchanges. Animal Behaviour, 59, 1127-1133.

Bourke, A. F. G. \& Franks, N. R. 1995. Social Evolution in Ants. Princeton, New Jersey: Princeton University Press.

Breed, M. D. 1998. Recognition pheromones of the honey bee. BioScience, 48, 463-470.

Breed, M. D. \& Bennett, B. 1987. Kin recognition in highly eusocial insects. In: Kin Recognition in Animals (Ed. by D. J. C. Fletcher \& C. D. Michener), pp. 243-285. New York: J. Wiley.

Breed, M. D., Diaz, P. H. \& Lucero, K. D. 2004. Olfactory information processing in honeybee, Apis mellifera, nestmate recognition. Animal Behaviour, 68, 921-928.

Buchwald, R. \& Breed, M. D. 2005. Nestmate recognition cues in a stingless bee, Trigona fulviventris. Animal Behaviour, 70, 1331-1337.

Buczkowski, G. \& Silverman, J. 2006. Geographical variation in Argentine ant aggression behaviour mediated by environmentally derived nestmate recognition cues. Animal Behaviour, 71, 327-335.

Cavalli-Sforza, L. L. \& Edwards, A. W. F. 1967. Phylogenetic analysis models and estimation procedures. Evolution, 21, 550-570.

Chapuisat, M., Goudet, J. \& Keller, L. 1997. Microsatellites reveal high population viscosity and limited dispersal in the ant Formica paralugubris. Evolution, 51, 475-482.

Chapuisat, M., Bocherens, S. \& Rosset, H. 2004. Variable queen number in ant colonies: no impact on queen turnover, inbreeding, and population genetic differentiation in the ant Formica selysi. Evolution, 58, 1064-1072. 
Chapuisat, M., Bernasconi, C., Hoehn, S. \& Reuter, M. 2005. Nestmate recognition in the unicolonial ant Formica paralugubris. Behavioral Ecology, 16, 15-19.

Crosland, M. W. J. 1990. The influence of the queen, colony size and worker ovarian development on nestmate recognition in the ant Rhytidoponera confusa. Animal Behaviour, 39, 413-425.

Crozier, R. H. \& Dix, M. W. 1979. Analysis of two genetic models for the innate components of colony odor in social Hymenoptera. Behavioral Ecology and Sociobiology, 4, 217-224.

Crozier, R. H. \& Pamilo, P. 1996. Evolution of Social Insect Colonies: Sex Allocation and Kin Selection. Oxford: Oxford University Press.

Dahbi, A., Hefetz, A., Cerda, X. \& Lenoir, A. 1999. Trophallaxis mediates uniformity of colony odor in Cataglyphis iberica ants (Hymenoptera, Formicidae). Journal of Insect Behavior, 12, 559-567.

Downs, S. G. \& Ratnieks, F. L. W. 1999. Recognition of conspecifics by honeybee guards uses nonheritable cues acquired in the adult stage. Animal Behaviour, 58, 643-648.

El Mousadik, A. \& Petit, R. J. 1996. High level of genetic differentiation for allelic richness among populations of the argan tree [Argania spinosa (L.) Skeels] endemic to Morocco. Theoretical and Applied Genetics, 92, 832-839.

Giraud, T., Pedersen, J. S. \& Keller, L. 2002. Evolution of supercolonies: the Argentine ants of southern Europe. Proceedings of the National Academy of Sciences, U.S.A., 99, 6075-6079.

Goudet, J. 1995. FSTAT (Version 1.2): a computer program to calculate F-statisics. Journal of Heredity, 86, 485-486.

Greenberg, L. 1979. Genetic component of bee odor in kin recognition. Science, 206, 1095-1097.

Hamilton, W. D. 1964. The genetical evolution of social behaviour. Journal of Theoretical Biology, 7, 1-52.

Hernandez, J. V., Goitia, W., Osio, A., Cabrera, A., Lopez, H., Sainz, C. \& Jaffe, K. 2006. Leaf-cutter ant species (Hymenoptera: Atta) differ in the types of cues used to differentiate between self and others. Animal Behaviour, 71, 945-952.

Hölldobler, B. \& Michener, C. D. 1980. Mechanims of identification and discrimination in social hymenoptera. In: Evolution of Social Behavior: Hypotheses and Empirical Tests (Ed. by H. Markl), pp. 35-58. Weinheim: Verlag Chemie.

Hölldobler, B. \& Wilson, E. O. 1977. The number of queens: an important trait in ant evolution. Naturwissenschaften, 64, 8-15.

Hölldobler, B. \& Wilson, E. O. 1990. The Ants. Berlin: Springer-Verlag.

Holway, D. A., Suarez, A. V. \& Case, T. J. 1998. Loss of intraspecific aggression in the success of a widespread invasive social insect. Science, 282, 949-952.

Holzer, B., Chapuisat, M., Kremer, N., Finet, C. \& Keller, L. 2006. Unicoloniality, recognition and genetic differentiation in a native Formica ant. Journal of Evolutionary Biology, 19, 2031-2039.

Howard, R. W. \& Blomquist, G. J. 2005. Ecological, behavioral, and biochemical aspects of insect hydrocarbons. Annual Review of Entomology, 50, 371-393.

Lahav, S., Soroker, V., Hefetz, A. \& Vander Meer, R. K. 1999. Direct behavioral evidence for hydrocarbons as ant recognition discriminators. Naturwissenschaften, 86, 246-249.

Langen, T. A., Tripet, F. \& Nonacs, P. 2000. The red and the black: habituation and the dear-enemy phenomenon in two desert Pheidole ants. Behavioral Ecology and Sociobiology, 48, 285-292.

Lenoir, A., Hefetz, A., Simon, T. \& Soroker, V. 2001. Comparative dynamics of gestalt odour formation in two ant species Camponotus fellah and Aphaenogaster senilis (Hymenoptera: Formicidae). Physiological Entomology, 26, 275-283.

Liang, D. \& Silverman, J. 2000. 'You are what you eat': diet modifies cuticular hydrocarbons and nestmate recognition in the Argentine ant, Linepithema humile. Naturwissenschaften, 87, 412-416.
Liebert, A. E. \& Starks, P. T. 2004. The action component of recognition systems: a focus on the response. Annales Zoologici Fennici, 41, 747-764.

Manly, B. F. J. 1997. Randomization, Bootstrap and Monte Carlo Methods in Biology. London: Chapman \& Hall.

Morel, L., Vander Meer, R. K. \& Lofgren, C. S. 1990. Comparison of nestmate recognition between monogyne and polygyne populations of Solenopsis invicta (Hymenoptera: Formicidae). Annals of the Entomological Society of America, 83, 642-647.

Ozaki, M., Wada-Katsumata, A., Fujikawa, K., Iwasaki, M., Yokohari, F., Satoji, Y., Nisimura, T. \& Yamaoka, R. 2005. Ant nestmate and non-nestmate discrimination by a chemosensory sensillum. Science, 309, 311-314.

Pamilo, P., Gertsch, P., Thorén, P. \& Seppä, P. 1997. Molecular population genetics of social insects. Annual Review of Ecology and Systematics, 28, 1-25.

Pirk, C. W. W., Neumann, P., Moritz, R. F. A. \& Pamilo, P. 2001. Intranest relatedness and nestmate recognition in the meadow ant Formica pratensis (R.). Behavioral Ecology and Sociobiology, 49, 366-374.

Reeve, H. K. 1989. The evolution of conspecific acceptance thresholds. American Naturalist, 133, 407-435.

Ross, K. G. 2001. Molecular ecology of social behaviour: analyses of breeding systems and genetic structure. Molecular Ecology, 10, 265-284.

Ruther, J., Sieben, S. \& Schricker, B. 2002. Nestmate recognition in social wasps: manipulation of hydrocarbon profiles induces aggression in the European hornet. Naturwissenschaften, 89, 111-114.

Satoh, T. \& Hirota, T. 2005. Factors affecting internest variation in the aggressiveness of a polygynous ant, Camponotus yamaokai. Entomological Science, 8, 277-281.

Schwander, T., Rosset, H. \& Chapuisat, M. 2005. Division of labour and worker size polymorphism in ant colonies: the impact of social and genetic factors. Behavioral Ecology and Sociobiology, 59, 215-221.

Sherman, P. W., Reeve, H. K. \& Pfennig, D. W. 1997. Recognition systems. In: Behavioural Ecology: an Evolutionary Approach (Ed. by J. R. Krebs \& N. B. Davies), pp. 69-96. Oxford: Blackwell Scientific.

Soroker, V., Vienne, C. \& Hefetz, A. 1995. Hydrocarbon dynamics within and between nestmates in Cataglyphis niger (Hymenoptera, Formicidae). Journal of Chemical Ecology, 21, 365-378.

Stuart, R. J. 1991. Nestmate recognition in Leptothoracine ants: testing for effects of queen number, colony size and species of intruder. Animal Behaviour, 42, 277-284.

Sundström, L. 1997. Queen acceptance and nestmate recognition in monogyne and polygyne colonies of the ant Formica truncorum. Animal Behaviour, 53, 499-510.

Tsutsui, N. D., Suarez, A. V. \& Grosberg, R. K. 2003. Genetic diversity, asymmetrical aggression, and recognition in a widespread invasive species. Proceedings of the National Academy of Sciences, U.S.A., 100, 1078-1083.

Vander Meer, R. K. \& Alonso, L. E. 2002. Queen primer pheromone affects conspecific fire ant (Solenopsis invicta) aggression. Behavioral Ecology and Sociobiology, 51, 122-130.

Vander Meer, R. K. \& Morel, L. 1998. Nestmate recognition in ants. In: Pheromone Communication in Social Insects (Ed. by R. K. Vander Meer, M. Breed, M. Winston \& K. E. Espelie), pp. 79-103. Boulder: Westview Press.

Wagner, D., Tissot, M., Cuevas, W. \& Gordon, D. M. 2000. Harvester ants utilize cuticular hydrocarbons in nestmate recognition. Journal of Chemical Ecology, 26, 2245-2257.

Waldman, B., Frumhoff, P. C. \& Sherman, P. W. 1988. Problems of kin recognition. Trends in Ecology \& Evolution, 3, 8-13.

Wilson, E. O. 1971. The Insect Societies. Cambridge, Massachusetts: Harvard University Press. 